\title{
Peripheral Blood Cytokines Levels and Post-Surgical Pain with Coated or Uncoated TKAs after 5 Years Follow-Up
}

\section{Raffaele Iorio, Edoardo Viglietta, Daniele Mazza, Fabio Marzilli*, Piergiorgio Drogo, Gerardo Salerno, Vincenzo Visco, Fabio Conteduca and Andrea Ferretti}

University of Rome "Sapienza", Sant'Andrea Hospital, Via di Grottarossa, Rome, Italy

*Corresponding Author: Fabio Marzilli, University of Rome "Sapienza", Sant'Andrea Hospital, Via di Grottarossa, Rome, Italy.
Received: February 272021

Published: March 19, 2021

(C) All rights are reserved by Fabio Marzilli., et al.

\begin{abstract}
Purpose: Twenty per cent of patients are estimated to be unsatisfied after total knee arthroplasty. Existence of an immune response against the implant has been recently investigated. This study aims to assess the cytokine patterns expressed in vivo in 5 years followup patients comparing uncoated and coated TKAs and correlating cytokines levels with post-surgical pain and clinical satisfaction. Methods: Fifty-eight TKA patients were retrospectively evaluated at a 5 years follow-up. Patients were divided in two groups according to the type of the implant. Thirty patients received a standard uncoated TKA and 28 subjects were treated with a titanium nitride (TiN) ceramic coated TKA. In each group, 10 patients were enrolled because they complained a post-surgical pain $>3$ at the Numerical Rating Scale (NRS) and were thus considered clinically unsatisfied. Peripheral blood samples were obtained and levels of inflammatory cytokines IL-4, IL-5, IL-6, Inf- $\gamma$ and TNF- $\alpha$ were analysed with a Luminex Magpix platform. Cytokines level was expressed as median fluorescent intensity (MFI).

Results: Comparison of the two groups showed significant higher TNF- $\alpha$ expression in the uncoated TKA group (P = 0.027). In both groups, a statistically higher IL- 6 and TNF- $\alpha$ levels were found in patients with post-surgical pain $>3$. Both for IL- 6 and TNF- $\alpha$, this difference was more significant in the uncoated $(\mathrm{P}=0.0007$ and $\mathrm{P}=0.0045)$ than in the coated group $(\mathrm{P}=0.044$ and $\mathrm{P}=0.049)$.

Conclusion: It is difficult to properly demonstrate an immunological response against the implant in TKA patients. In our study, higher cytokines expression was found with standard uncoated implants and in painful-unsatisfied patients. Further evidences, are needed to establish if an immune mechanism may be responsible for cases of unexplained poor functioning TKAs.
\end{abstract}

Keywords: Unsatisfied; Pain; TKA; Metal Allergy; Hypersensitivity

\section{Introduction}

Total Knee Arthroplasty (TKA) represents a successful treatment in degenerative and inflammatory knee diseases, providing improvement of pain, function and daily life in most patients at long-term follow-up [1]. However, not all patients experience such excellent clinical results [2]. Twenty per cent of patients are esti- mated to be unsatisfied $[3,4]$ and $2 \%$ of TKAs are estimated to need revision surgery after 5 or more years [1]. Periprosthetic joint infections (PJIs), instability, aseptic loosening or periprosthetic fractures are common causes of implant failure or painful TKA and can be easily recognised [5]. Recently, several studies proposed metal hypersensitivity as a supposed mechanism for poor functioning TKAs in the absence of other clear causes [6-11]. 
Evidence of an immunological reaction to metal, urological, cardiovascular, plastic or dental devices have been reported in the literature [12-15]. Also for orthopaedic implants the existence of an immune response has been investigated. In the general population, the prevalence of metal hypersensitivity is about 15\% [15]. This prevalence is higher in patients with TKA and even greater in patients with malfunctioning implants, $25 \%$ and $60 \%$, respectively [15]. Thus, hypoallergenic prostheses were recently proposed. However, doubts are still present about the existence and the findings of immune response to the prosthesis and the diagnostic relevance of common diagnostic tests, i.e. patch test or lymphocyte proliferation test (LTT), is still unclear. Lutzner., et al. [16] did not find an increase of metal sensitisation rate in TKA patients one year after surgery. This suggests that skin and periprosthetic reactions due to metal hypersensitivity could involve different immunological mechanisms. Furthermore, a clear correlation with clinical outcomes is still not defined.

Recently, the role of innate immunity response to metal implants was underlined [9] and the cytokines pattern associated was investigated $[17,18]$. Cytokines production is believed to stimulate osteoclast activation, leading to osteolysis and implant failure $[6,15,19,20]$. However, no study correlating cytokine expression with clinical outcomes in different types of implant is present.

\section{Aim of the Study}

The aim of this study is to assess the cytokine patterns expressed in vivo in 5 years follow-up patients comparing uncoated and coated TKAs and to correlate cytokines levels with post-surgical pain and clinical satisfaction. Our hypotheses are that blood cytokines expression may correlate with clinical satisfaction referred by patients and that difference in cytokines levels is present with coated and uncoated TKAs, due to a different capability to determine an immune response against prostheses themselves.

\section{Methods}

\section{Study population}

In this study, 219 TKAs (216 patients) performed in our centre between February 2012 and December 2013 were included. All patients were retrospectively evaluated at a midterm follow-up of not less than 5 years.

Exclusion criteria were represented by underlying conditions altering immune system such as a previously diagnosed metal al- lergy, chronic inflammatory diseases, autoimmune disease, tumour in previous 5 years or current immunosuppressive therapies. Additionally, in order to eliminate confounding factors, BMI > 35, cemented TKA, contralateral TKA and presence of other metal implants were exclusion criteria.

Among the remaining 116 patients, 20 subjects were selected because they were clinically unsatisfied complaining knee pain during daily activity and 38 satisfied subjects were enrolled as control. Common causes of implant failure and consequently postsurgical pain (i.e. instability, PJIs, aseptic loosening -due to the impossibility, to date, to diagnose which were due to allergy and which not- or periprosthetic fractures) were excluded.

Thus, patient were divided in two groups according to the type of implant. Thirty patients who received a standard uncoated TKA (The LCS ${ }^{\circledR}$ COMPLETE Knee System, DePuy Synthes, Warsaw, IN) were included in the LCS group: 10 painful unsatisfied and 20 painless satisfied patients. On the other side, 28 patients treated with a titanium nitride (TiN) ceramic coated TKA (The TiN Ceramic UltraCoat ${ }^{\circledR}$ Coating, Endotec, South Orange, NJ) were included in the Endotec group: 10 painful unsatisfied and 18 painless satisfied patients. Selection of patients was accurately performed in order to obtain homogeneity into the two groups (Figure 1). Both types of TKA were not cemented.

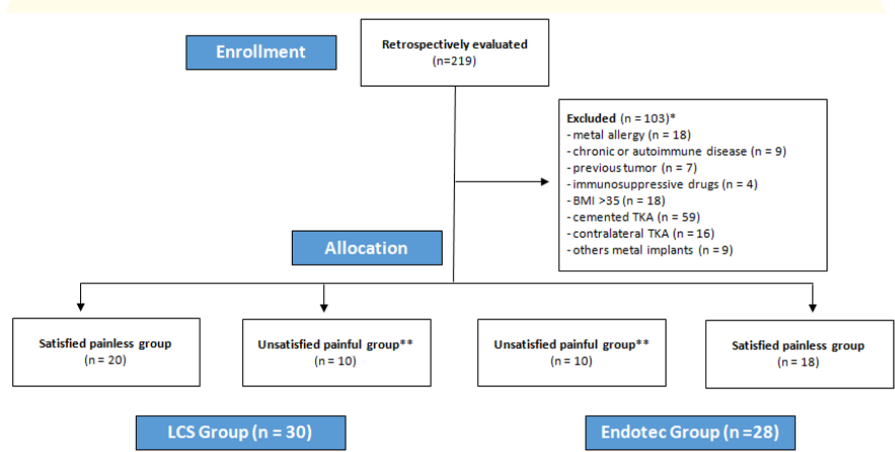

Figure 1: Patients selection. Both the LCS and the Endotec group included 10 painful unsatisfied patients and a control group of 20 and 18 satisfied patients respectively.

*: Note that 103 patients were excluded but more exclusion criteria could be present in a single patient simultaneously.

**: Instability, malalignment, aseptic loosening and periprosthetic fracture were thoroughly ruled out. 
All patients gave written informed consent to enrolment in the study, which was approved by the Institutional Ethics Committee.

\section{Clinical and radiological evaluation}

The Numerical Rating Scale (NRS) was administered both at rest and after five-minutes walking to assess level of satisfaction. NRS is a subjective unimodal measure in which patients rate their pain on an eleven-point numerical scale. A score higher than three (NRS > 3), at rest or during walking test, was used to select patients to be included as painful unsatisfied prostheses.

Periprosthetic fractures, radiolucencies, aseptic failures and malalignment were excluded by standard weight-bearing knee radiographs in antero-posterior and latero-lateral views of the knee. In cases of suspicion, joint aspiration was performed and laboratory exams (i.e. microbiological cultures, white blood cells, marker of inflammation, etc) were conducted to exclude PJIs. Common causes of pain and malfunctioning TKA were thus investigated.

\section{Peripheral blood cytokine expression}

Blood samples were obtained from each patient through a peripheral venipuncture and collected in EDTA tubes. Serum samples obtained from each patient were immediately centrifugated at $4000 \mathrm{rpm}$ for 10 minutes and aliquots of serum were stored at $-20{ }^{\circ} \mathrm{C}$ until assessed. Each sample was evaluated for the presence and concentration of inflammatory cytokines IL-4, IL-5, IL-6, Interferon Gamma (Inf- $\gamma$ ) and Tumor Necrosis Factor Alfa (TNF- $\boldsymbol{\alpha}$ ). The cytokines were analysed with a Luminex Magpix platform expressing the level of molecules as median fluorescent intensity (MFI).

\section{Statistics}

All statistical analyses were performed using R Version 3.5.3 (2019-03-11) - 'GREAT TRUTH' COPYRIGHT @ 2019. The R (FOUNDATION FOR STATISTICAL COMPUTING) was employed for all analyses. If the data followed the normal distribution, statistical analyses was performed by the D'Agostino Pearson normality test. The non-parametric Mann-Whitney $U$ test was used to examine the difference between the two groups as the data were not normally distributed. A P-value $<0.05$ was considered statistically significant.

\section{Results}

Fifty-eight patients were finally enrolled in the study. In the LCS group, the mean follow-up was $70.9 \pm 6.9$ (range: 60 - 85) months. Eleven patients (37\%) had involvement of the right knee, while 19 $(67 \%)$ had involvement of the left. The patient population included $11(37 \%)$ males and 19 (63\%) females, with a mean age at surgery of $70.5 \pm 6.9$ years (range $54-85$ ). The mean follow-up of the Endotec group was $68.9 \pm 4.6$ (range: 60 - 81) months. The right knee was involved in 13 (46\%) patients and the left knee in 15 (54\%). The patient population included 8 (29\%) males and 20 (71\%) females, with a mean age at surgery of $68.5 \pm 10.1$ years (range 46 86). Ten patients complained of pain in both groups. Demographic data and homogeneity of the two groups are reported in table 1.

\begin{tabular}{|c|c|c|}
\hline & ENDOTEC & LCS \\
\hline Patients & 28 & 30 \\
\hline Females (n/\%) & $20 / 71 \%$ & $19 / 63 \%$ \\
\hline Right Side (n/\%) & $13 / 46 \%$ & $11 / 37 \%$ \\
\hline Age (mean \pm SD) & $68,5 \pm 10,1$ & $70,5 \pm 6,9$ \\
\hline BMI (mean \pm SD) & $29,9 \pm 2,8$ & $29,2 \pm 3,3$ \\
\hline Follow up (mean \pm SD) & $68,9 \pm 4,6$ & $70,9 \pm 6.9$ \\
\hline
\end{tabular}

Table 1: Demographic data of the study population. No statistical differences were present for any parameter $(p>0.05)$. Data were expressed by absolute number (n) and percentage (\%) or by mean \pm standard deviation (SD) as indicated in the left column.

In both groups, levels of IL-4, IL-5, IL-6, Inf- $\gamma$ and TNF- $\alpha$ were evaluated. The overall comparison of the two groups showed significant higher TNF- $\alpha$ expression in the LCS group (LCS group MFI: 7.25 (range 3.75-12) vs Endotec group MFI: 5.5 (range 3.5-6.5); P = 0.027). No statistical differences were found for IL-4, IL-5, IL-6 and Inf- $\gamma$. Overall comparison is reported in figure 2 and table 2.

Secondly, a correlation between cytokines levels and patients satisfaction in term of post-surgical pain was investigated. In each group a comparison between patients who noted pain (NRS > 3 ) and patients without pain (NRS $=0-3$ ) was conducted. In LCS group, a statistically higher level of IL-6 (painful TKA MFI: 16 


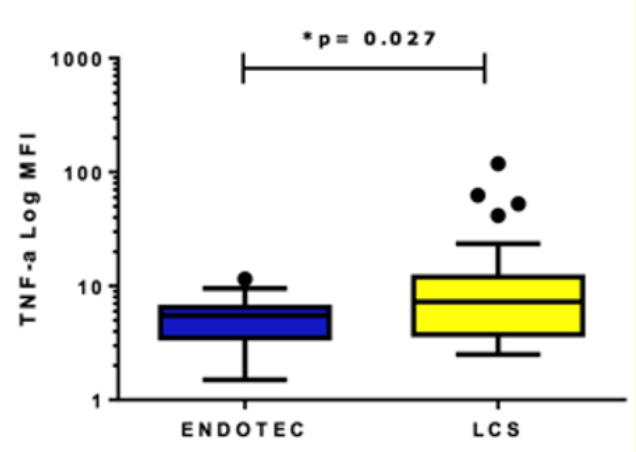

Figure 2: Overall comparison of TNF- $\alpha$ levels between Endotec and LCS groups. A significant higher TNF- $\alpha$ expression was present in the LCS group.

\begin{tabular}{|c|c|c|c|}
\hline & ENDOTEC MFI (range) & LCS MFI (range) & P-value \\
\hline TNF- $\alpha$ & $5.5(3.5-6.5)$ & $7.25(3.75-12)$ & 0.027 \\
\hline IL-4 & $0(0-2)$ & $0(0-1.74)$ & 0.82 \\
\hline IL-5 & $0(0-2.5)$ & $0(0-2)$ & 0.76 \\
\hline IL-6 & $10.5(6.5-19.5)$ & $6.5(4.75-9.25)$ & 0.21 \\
\hline Inf- $\gamma$ & $4.25(3.5-8)$ & $5.5(4-8.75)$ & 0.13 \\
\hline
\end{tabular}

Table 2: Overall comparison of the LCS and Endotec groups. Cytokines levels are expressed as median fluorescent intensity (MFI) and range.

(range 13 - 24.75) vs painless TKA MFI: 6 (range 4.25 - 9.75)) and TNF- $\alpha$ (painful TKA MFI: 24.5 (range 5.25 - 76.5) vs painless TKA MFI: 6.5 (range 3.5 - 9.5)) was found in the 10 patients with pain than in those without. Also, in Endotec group a statistically higher level of IL-6 (painful TKA MFI: 34.5 (range 13.5 - 130.5) vs painless TKA MFI: 10.5 (range 5 - 13)) and TNF- $\alpha$ (painful TKA MFI: 11.5 (range 4 - 46) vs painless TKA MFI: 5.5 (range 3.5 - 7.5)) was found in the 10 patients with pain. The difference was more significant in the LCS group than in the Endotec group both for IL- 6 and TNF- $\alpha$ (P $=0.0007$ and $\mathrm{P}=0.0045$ vs $\mathrm{P}=0.044$ and $\mathrm{P}=0.049$ respectively). No difference was found for IL-4, IL-5 and Inf- $\gamma$. Comparisons are shown in figure 3 and table 3.
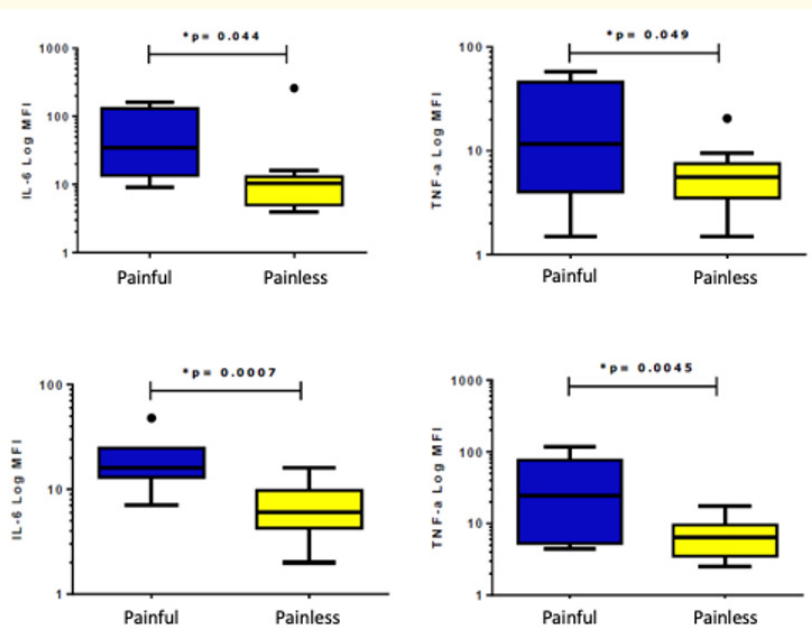

Figure 3: TNF- $\alpha$ and IL-6 levels related to clinical outcomes. A statically higher expression of IL- 6 and TNF- $\alpha$ was present in painful prosthesis both in the Endotec (upper image) and LCS group (lower image).

\begin{tabular}{|c|c|c|c|c|}
\hline & & Painful MFI (range) & $\begin{array}{c}\text { Painless MFI } \\
\text { (range) }\end{array}$ & P-value \\
\hline \multirow{2}{*}{ Enodotec } & TNF- $\alpha$ & $11.5(4-46)$ & $5.5(3.5-7.5)$ & 0.049 \\
\cline { 2 - 5 } & IL-6 & $\begin{array}{c}34.5 \\
(13.5-130.5)\end{array}$ & $10.5(5-13)$ & 0.044 \\
\hline \multirow{2}{*}{ LCS } & TNF- $\alpha$ & $\begin{array}{c}24.5 \\
(5.25-76.5)\end{array}$ & $6.5(3.5-9.5)$ & 0.0045 \\
\cline { 2 - 5 } & IL-6 & $16(13-24.75)$ & $6(4.25-9.75)$ & 0.0007 \\
\hline
\end{tabular}

Table 3: Cytokines levels related to clinical satisfaction in Endotec and LCS group. Cytokines levels are expressed as median fluorescent intensity (MFI) and range. Painful prosthesis were considered for a NRS score $>3$.

\section{Discussion and Conclusion}

The main finding of our study is that after a minimum of 5 years follow-up cytokines levels were found to be higher with uncoated TKA and in painful unsatisfied patients. Our hypotheses that cy- 
tokines expression correlates with clinical satisfaction and type of the implant have been confirmed.

TKA represents a successful treatment with a high grade of clinical satisfaction. However, in some cases, post-surgical pain limits the performance of the implant. The real relevance of this complication is not clearly defined $[3,21,22]$. According to Wylde., et al. [23], 44\% of patients suffer from various persistent grades of post-surgical pain after TKA. Patients' satisfaction is a primary goal in joint arthroplasty and persistent post-surgical pain may be considered as failure of the implant. When the most common causes are excluded, it is difficult to identify the origin and the solution of the matter.

Recently, metal hypersensitivity has been proposed to explain the undefined cases of painful prosthesis. Several studies were performed to assess the role of an immune metal hypersensitivity in a painful and poorly functioning prosthesis. However, the exact immunological mechanism is still not clear and the proper treatment for suspected metal allergy is ambiguous [6,17]. In 2012, metal hypersensitivity was reported as being responsible for $5.9 \%$ of all revisions for hip arthroplasty and for $1.3 \%$ of TKA revisions [4]. Nevertheless, TKA is more commonly associated with malfunction or poor outcomes of the implant, being reported a likely $20 \%$ of unsatisfied patients.

As for skin metal allergy, also for prosthetic complications has been supposed that metal ion released from the prosthesis could activate the immune system [15,20]. Caicedo., et al. [24] in a retrospective study, reported that patients with a painful Total Joint Arthroplasty (TJA) exhibited a higher metal sensitisation than a control group of subjects without TJA or pain. Furthermore, among patients with idiopathic post-surgical pain, females showed a statistically significant higher metal hypersensitivity.

Nickel is universally accepted as the most common sensitiser in humans followed by cobalt and chromium [9,25]. Thus, nickelfree hypoallergenic prostheses were produced in order to avoid the consequences of a local immune response to the implant. Also, other components of the prosthesis, such as plastic polyethylene or cement polymer of polymethyl methacrylate (PMMA) can be possible sensitisers [26].
However, differences between the well-known cell-mediated delayed-type hypersensitivity (Type IV hypersensitivity) of skin metal allergy and the immune response against non-self components of the prosthesis in the joint environment are supposed to exist [6]. In fact, patch test and LTT, commonly used in diagnosing metal hypersensitivity [27], reported ambiguous results in painful TKA patients [6]. Bravo., et al. [28] recently reported that no correlation between a positive patch test and an increased risk for TKA failure exists. Different results were reported by Granchi., et al. [29] who showed that the risk of failure is fourfold more likely in patients with preoperative findings of metal allergy. However, no statistical difference of patch test was found between chromiumcobalt based and titanium-based TKAs.

Even if the exact mechanism remains unexplained, the immune system is likely to be involved in the poor functioning TKAs when the most common causes have been excluded. Recently, cytokines level was measured trying to elucidate the immunological mechanism behind. Additionally, the role of a specific cytokine pattern in persistent post-surgical pain has recently been demonstrated [3]. Hallab., et al. [9] in a review from 2017, underlined the importance of cytokines and chemokines based innate responses of the immune system accountable for the implant failure. They found that the most important mediators involved are TNF- $\alpha$, IL-1 $\beta$ and IL-6, together with chemokines such as Monocyte Chemotactic Protein-1 (MCP-1). They also underlined the necessity of in vivo studies to better understand and confirm the results of commonly performed in vitro studies.

For these reasons, we selected cytokines of the innate immune response (i.e. IL- 4 , IL-5, IL- 6 , Inf- $\gamma$ and TNF- $\alpha$ ) released by innate immune cells. We believed that cytokines pattern, measured in patients' peripheral blood, could be an in vivo expression of the immunologic response against implant. To demonstrate that, difference in cytokine expression must be present according to the type of implant and mostly according to clinical satisfaction.

Cytokine patterns in peripheral blood have yet been described to reflect local tissue inflammation in various conditions (e.g. rheumatoid arthritis, malignant tumour, bowel inflammatory disease) [30]. Furthermore, Teo., et al. [10] investigated the possible role 
of inflammatory cytokines in post-surgical pain. A correlation between IL-6 and TNF- $\alpha$ level and post-surgical pain was found. They also reported 3 years as being necessary to generate an immune response to the implant. Thus, we retrospectively selected patients at a minimum 5 years follow-up.

In both groups, comparing satisfied and unsatisfied (painless vs painful) patients, statistically significant differences were found in IL- 6 and TNF- $\alpha$ levels. The significantly higher cytokine expression in patients with a painful prosthesis could be a substantial proof of immunological involvement in clinical outcomes. This higher expression in painful patients was found to be even greater in the LCS group ( $\mathrm{P}=0.0007$ and 0.0045 for IL- 6 and TNF- $\alpha$, respectively) than in Endotec group and it is likely related to obvious higher concentration of metal particles in standard TKAs. The reason why an immune, clinical related, response was also found in the Endotec group could be explained by the small amount of metal ions present in coated implants. Although is not possible to say with certainty, this small presence has been postulated to be enough to generate a similar, but lower immune response [11].

Results of the overall comparison between the two groups showed a higher TNF- $\alpha$ level in the LCS group than in the Endotec group ( $\mathrm{P}=0.027$ ). This result suggests that a standard uncoated TKA could stimulate an immune response more than a coated implant. Similar levels of the other cytokines tested were found in the two groups. The absence of a difference in IL- 6 levels could be due to the majority of satisfied patients in both groups, likely able to mitigating possible differences between coated or uncoated implants. The lack of a further comparison of cytokines levels in painful patients between the LCS and the Endotec group represents a well known limitation of the study.

To the best our knowledge, this is the first study which compares clinical satisfaction and cytokine levels as indicators of an immunologic involvement in TKAs outcome. The cytokines pattern in the peripheral blood of patients with a standard uncoated or a surface coated TKA were recently evaluated by Thomas., et al [18]. IL-6, IL-8 and IL-10 levels were statistically higher in the uncoated implant patients compared both with coated TKA patients and with a healthy control group. They reported that the results were not affected by the inclusion of patients with a dermatologic metal allergy history in the control group. In fact, their cytokine levels were similar to patients without allergy. This could be further evidence that, between skin and prosthetic metal allergy, differences do exist. Their results are consistent with the higher IL-6 and TNF- $\alpha$ levels found in our study. However, in their evaluation, they included all patients with good clinical outcomes, so is not possible to conclude if the cytokines increase may have a relevant clinical correlation or not. We did not evaluate IL-8 because the role of chemoattractant mediators was not investigated in our study.

Local cytokines pattern present in the periprosthetic tissue was also studied [17]. In TKA patients undergoing revision surgery for unexplained complications like pain, effusion or reduced range of motion, the levels of IFN- $\gamma$, TGF- $ß$ and IL- 6 were upregulated compared to the control group of patients without implant.

The study has some limitations 1) The absence of preoperative cytokine expression. However, the exclusion criteria adopted mitigate this limitation. In fact, by excluding patients with other allergic, autoimmune, inflammatory or neoplastic diseases and with previous metal implants, the most common confounding factors interacting with the immune system were eliminated. Additionally, common cause of painful or malfunctioning TKA have been excluded, allowing not to attribute cytokine expression to other causes. 2) A clinical evaluation of all patient undergoing TKA in the study period was not performed, thus it is not possible to say if one implant globally presents better clinical outcomes than the other. However, we voluntarily selected two homogeneous groups (10 unsatisfied patients and about twice patients with good clinical outcomes), because the aim of this study was not to asses which implant is better, but which one could have a stronger immunogenic role. 3) Patch test was not performed to determine metal sensitisation. However, we do not believe that patch test results have a defined correlation with the immune response in TKAs. 4) The small study population. However, this is due to the study design. Furthermore, it is consistent with other studies present in the literature 5) The lack of a comparison of painful patients between the LCS and the Endotec group.

Strengths of this study consist in: 1) Both types of prosthesis are cementless, thus eliminating interference of PMMA in the in- 
terpretation of results. 2) The 5 years follow-up is long enough to generate an immune response against the implant. 3) This is an in vivo evaluation of the immunological response against uncoated or coated TKAs and few studies with the same characteristics are present in literature. 4) Lastly, this is the first study which makes a comparison between cytokine expression and the clinical satisfaction perceived by patients.

It is difficult to clearly demonstrate an immunological response against the implant in TKA unsatisfied patients. Cytokines levels found in our study were higher with standard uncoated implants and in painful-unsatisfied patients. It is not possible to state surely but the cytokines expression found may be evidence of an immune involvement in TKA patients. This involvement seems to affect the level of patients post-surgical pain and consequently satisfaction and it seems to be different according to the type of implant.

The proper relation between orthopedic implants and immune mediators may help in understanding cases of unexplained implant pain after TKA. However, exact significance of this immunological involvement needs further studies to be better understood.

\section{Disclosure}

On behalf of all authors, the corresponding author states that:

- $\quad$ All authors listed meet the authorship criteria according to the latest guidelines of the International Committee of Medical Journal Editors.

- $\quad$ All authors are in agreement with the manuscript.

- $\quad$ There is no conflict of interest.

- $\quad$ No financing was received.

\section{Bibliography}

1. Kane RL., et al. "Total knee replacement". Evidence Report/ Technology Assessment 86 (2003): 1-8.

2. Beverland D. "Patient satisfaction following TKA: Bless them all!" Orthopedics 33.9 (2010): 657.

3. Grosu I., et al. "Pain after knee arthroplasty: an unresolved issue". Knee Surgery, Sports Traumatology, Arthroscopy 22.8 (2014): 1744-1758.
4. Gunaratne R., et al. "Patient Dissatisfaction Following Total Knee Arthroplasty: A Systematic Review of the Literature". The Journal of Arthroplasty 32.12 (2017): 3854-3860.

5. Carulli C., et al. "Painful knee arthroplasty: definition and overview". Clinical Cases in Mineral and Bone Metabolism 8.2 (2011): 23-25.

6. Carossino AM., et al. "Hypersensitivity reactions to metal implants: laboratory options". BMC Musculoskeletal Disorders 17.1 (2016): 486.

7. Middleton S and Toms A. "Allergy in total knee arthroplasty: a review of the facts". The Bone and Joint Journal 98-B.4 (2016): 437-441.

8. Akil S., et al. "Metal hypersensitivity in total hip and knee arthroplasty: Current concepts". Journal of Clinical Orthopaedics and Trauma 9.1 (2018): 3-6.

9. Hallab NJ and Jacobs JJ. "Chemokines Associated with Pathologic Responses to Orthopedic Implant Debris". Frontiers in Endocrinology 8 (2017): 5.

10. Teo WZW and Schalock PC. "Hypersensitivity Reactions to Implanted Metal Devices: Facts and Fictions". The Journal of Investigational Allergology and Clinical Immunology 26.5 (2016): 279-294.

11. Delimar D., et al. "Orthopedic and Cutaneous Reactions to Nickel after Total Hip Replacement". Acta Dermatovenerologica Croatica 26.1 (2018): 39-43.

12. Almpanis GC., et al. "Nickel allergy, Kounis syndrome and intracardiac metal devices". International Journal of Cardiology 145.2 (2010): 364-365.

13. Thomas P. "Impaired fracture healing and eczema from titanium based osteosynthesis with corresponding T-cell hyperresponsiveness in vitro: A case of titanium hypersensitivity?" Contact Dermatitis 55 (2006): 199-202.

14. Yamauchi R., et al. "Pacemaker dermatitis from titanium". Contact Dermatitis 42 (2000): 52-53. 
15. Hallab N., et al. "Metal sensitivity in patients with orthopaedic implants". Journal of Bone and Joint Surgery American 83.3 (2001): 428-436.

16. Lützner J., et al. "Metal hypersensitivity and metal ion levels in patients with coated or uncoated total knee arthroplasty a randomised controlled study". International Orthopaedics 37.10 (2013): 1925-1931.

17. Thomas P., et al. "Patients with intolerance reactions to total knee replacement: combined assessment of allergy diagnostics, periprosthetic histology, and peri-implant cytokine expression pattern". BioMed Research International (2015): 910156.

18. Thomas P., et al. "Blood cytokine pattern and clinical outcome in knee arthroplasty patients: comparative analysis 5 years after standard versus "hypoallergenic" surface coated prosthesis implantation". Acta Orthopaedica 89.6 (2018): 646-651.

19. Hallab NJ and Jacobs JJ. "Biologic effects of implant debris". Bulletin of the Hospital for Joint Diseases 67 (2009): 182-188.

20. Savarino L., et al. "Effects of metal ions on white blood cells of patients with failed total joint arthroplasties". Journal of Biomedical Materials Research 47.4 (1999): 543-550.

21. Beswick AD., et al. "What proportion of patients report longterm pain after total hip or knee replacement for osteoarthritis? A systematic review of prospective studies in unselected patients". BMJ Open 2 (2012): e00043518.

22. Piscitelli P., et al. "Painful prosthesis: approaching the patient with persistent pain following total hip and knee arthroplasty". Clinical Cases in Mineral and Bone Metabolism 10.2 (2013): 97-110.

23. Wylde V., et al. "Persistent pain after joint placement: prevalence, sensory qualities, and post-operative determinants". Pain 152 (2011): 566-572.
24. Caicedo MS., et al. "Females with Unexplained Joint Pain Following Total Joint Arthroplasty Exhibit a Higher Rate and Severity of Hypersensitivity to Implant Metals Compared with Males: Implications of Sex-Based Bioreactivity Differences". Journal of Bone and Joint Surgery American 99.8 (2017): 621628.

25. Haudrechy P., et al. "Nickel release from nickel-plated metals and stainless steels". Contact Dermatitis 31 (1994): 249-255.

26. Schuh A., et al. "Allergic reaction to components of bone cement after total knee arthroplasty". Zentralbl Chir 131 (2006): 429-431.

27. Fisher DA., et al. "Looks good but feels bad: factors that contribute to poor results after total knee arthroplasty". The Journal of Arthroplasty 22.26 (2007): 39-42.

28. Bravo D., et al. "No increased risk of knee arthroplasty failure in patients with positive skin patch testing for metal hypersensitivity: a matched cohort study". The Journal of Arthroplasty 31.8 (2016): 1717-1721.

29. Granchi D., et al. "Sensitivity to implant materials in patients with total knee arthroplasties". Biomaterials 29.10 (2008): 1494-1500.

30. Tan Y., et al. "Cytokine Imbalance as a Common Mechanism in Both Psoriasis and Rheumatoid Arthritis". Mediators of Inflammation (2017): 2405291.

\section{Assets from publication with us}

- Prompt Acknowledgement after receiving the article

- Thorough Double blinded peer review

- Rapid Publication

- Issue of Publication Certificate

- High visibility of your Published work

Website: www.actascientific.com/

Submit Article: www.actascientific.com/submission.php

Email us: editor@actascientific.com

Contact us: +919182824667 